\title{
NÍVEL DE ATIVIDADE FÍSICA, DOR E EDEMA E SUAS RELAÇÕES COM A DISFUNÇÃO MUSCULAR DO JOELHO DE IDOSOS COM OSTEOARTRITE
}

\author{
Zacaron KAM ${ }^{1,2}$, Dias JMD ${ }^{3}$, Abreu NS ${ }^{1,2}$ E Dias RC ${ }^{3}$ \\ ${ }^{1}$ Curso de Fisioterapia, Faculdade de Ciências Médicas e da Saúde de Juiz de Fora, Juiz de Fora, MG - Brasil \\ ${ }^{2}$ Programa de Pós-Graduação em Ciências da Reabilitação, Universidade Federal de Minas Gerais - UFMG, Belo \\ Horizonte, MG - Brasil \\ ${ }^{3}$ Departamento de Fisioterapia, UFMG, Belo Horizonte, MG - Brasil \\ Correspondência para: Katy Andrade Monteiro Zacaron, Rua da Bahia, 258, Poço Rico, CEP 36020-080, Juiz de Fora , \\ MG - Brasil, e-mail: katyzac@powermail.com.br
}

Recebido: 30/06/2005 - Aceito: 22/03/2006

\begin{abstract}
RESUMO
Contextualização: O decréscimo da função dos músculos quadríceps (Q) e isquiotibiais (IT) apresenta-se potencializado na população idosa com osteoartrite (OA) de joelhos, podendo ser atribuído ao menor nível de atividade física (NAF) ou à dor e ao edema. Objetivos: Comparar o desempenho de Q e IT de idosos assintomáticos e com OA de joelhos e correlacionar tal desempenho com a dor e o edema. Métodos: A amostra constitui-se de quinze idosos com OA de joelho bilateral (grupo OA) e quinze assintomáticos (grupo AS). O dinamômetro isocinético, Biodex System 3-Pro, foi utilizado para avaliar a performance muscular e a escala visual análoga (EVA) numérica para registro da dor. O edema foi avaliado por teste manual. Teste t-Student foi empregado para comparar as variáveis demográficas, antropométricas, trabalho de Q (TRAB Q), de IT (TRAB IT) e Relação IT/Q. Teste Qui-quadrado foi utilizado para a comparação do NAF e o coeficiente de Spearman para verificar correlação entre as variáveis edema e dor com TRAB Q, TRAB IT e Relação IT/Q. Resultados: Não houve diferença estatisticamente significante entre as variáveis antropométricas, demográficas e $\operatorname{NAF}(p>0,05)$. TRAB $Q$ apresentou-se estatisticamente menor $(\mathrm{p}=0,001)$ no grupo OA e a Relação IT/Q, mostrou-se estatisticamente maior neste grupo $(\mathrm{p}=0,04)$. Não houve correlação entre as variáveis dor e edema com TRAB Q, TRAB IT e Relação IT/Q (rho $\leq-0,468 ; p \geq 0,229$ ). Conclusão: A redução da função muscular dos idosos com OA de joelhos não pode ser atribuída unicamente a um menor NAF desta população.
\end{abstract}

Palavras-chave: idosos, osteoartrite de joelho, atividade física, dor, edema, disfunção muscular.

\section{ABSTRACT \\ Physical Activity Levels, Pain and Swelling and Their Relationships with Knee Muscle Dysfunction in Elderly People with Osteoarthritis}

Background: Decreased quadriceps and hamstring muscle function appears to be greater in the elderly population with knee osteoarthritis. This may be due to lower physical activity levels or to pain and swelling. Objective: To compare quadriceps and hamstring performance in elderly people with and without knee osteoarthritis and to correlate this with pain and swelling. Method: The sample was composed of 15 elderly people with bilateral knee osteoarthritis (osteoarthritis group) and 15 asymptomatic elderly people (asymptomatic group). The Biodex System 3-Pro ${ }^{\circledast}$ isokinetic dynamometer was used to evaluate muscle performance, and a visual analog scale (VAS) to register the pain. The swelling was assessed by a manual test. Student's test was applied to compare the demographics, anthropometric variables, quadriceps work, hamstring work and hamstring/quadriceps ratio. The chi-squared test was used to compare physical activity levels and Spearman coefficients to verify correlations between the swelling and pain variables and the quadriceps work, hamstring work and hamstring/quadriceps ratio. Results: No statistically significant differences were detected between the anthropometric variables, demographics and physical activity levels $(p>0.05)$. Quadriceps work was statistically smaller $(\mathrm{p}=0.001)$ in the osteoarthritis group and the hamstring/quadriceps ratio was statistically larger in this group $(p=0.04)$. There was no correlation between the pain and swelling variables and the quadriceps work, hamstring work and hamstring/quadriceps ratio (rho $\leq-0.468 ; p \geq 0.229$ ). Conclusion: The reduced muscle function among elderly people with knee osteoarthritis cannot solely be attributed to the lower physical activity levels of this population.

Key words: elderly people, knee osteoarthritis, physical activity, pain, swelling, muscle dysfunction. 


\section{INTRODUÇÃO}

A osteoartrite (OA) é uma doença articular crônicodegenerativa que evidencia desgaste da cartilagem articular, na qual, dentre as articulações de sustentação de peso, o joelho é o mais freqüentemente afetado ${ }^{1}$.

Clínica e radiograficamente, a OA caracteriza-se por dor, rigidez matinal, crepitação óssea, atrofia muscular, estreitamento de espaço intra-articular, formações osteofíticas, esclerose do osso subcondral e formações císticas².

Embora as pesquisas tenham pouco sucesso em elucidar a patogênese da OA de joelho, alguns fatores de risco para esta doença encontram-se bem estabelecidos. Por manifestarse em cerca de $50 \%$ dos idosos com mais de 65 anos e em $80 \%$ dos acima de 75 anos, o envelhecimento inclui-se entre estes fatores ${ }^{3}$. Além do envelhecimento, a obesidade, lesões ou cirurgias prévias, esforço ocupacional ou recreacional cumulativo, mau alinhamento articular e fraqueza muscular são alguns dos fatores que também predispõem à $\mathrm{OA}^{2,3}$.

O desempenho muscular apresenta um decréscimo com o processo de envelhecimento ${ }^{4}$, sendo a sarcopenia causa direta da redução da força muscular no idoso ${ }^{4,5}$. O fenômeno da sarcopenia caracteriza-se pela redução no número, no tamanho e no nível da vascularização das fibras musculares ${ }^{4}$.

Conjuntamente à sarcopenia, a perda de força muscular que acompanha o processo de envelhecimento, e também, é atribuída a fatores como a alteração da composição química das fibras musculares, redução no número e tamanho de motoneurônios e consequentemente das unidades motoras ${ }^{6}$, da fragmentação e degeneração das junções neuromusculares, além do desalinhamento dos elementos pré e pós-sinápticos ${ }^{6}$.

O decréscimo da função dos músculos quadríceps $(\mathrm{Q})$ e isquiotibiais (IT) apresenta-se potencializada na população idosa portadora de OA de joelhos ${ }^{7,8}$, podendo ser atribuída ao fator comportamental caracterizado por menor nível de atividade física adotada por esta população ${ }^{8}$ ou aos sinais e sintomas clínicos inerentes à doença ${ }^{7,9}$.

A dor e o fluido intra-articular excessivo, que são comuns em afecções articulares como a OA, sensibilizam os mecanorreceptores capsulares que emitem sinais para interneurônios inibitórios medulares os quais inibem os motoneurônios Alfa e, consequentemente, os sinais que seriam transmitidos ao quadríceps ${ }^{9,10}$. Este fenômeno é denominado inibição muscular artrogênica (IMA) e é, provavelmente, gerado pela informação aferente anormal que parte da articulação afetada resultando em ativação diminuída dos músculos que agem nesta articulação $0^{9,10}$.

Em virtude do fato de o decréscimo na função muscular dos idosos com e sem OA de joelhos ser um fator limitante da capacidade funcional desta população e, consequentemente, da independência física ${ }^{11}$, torna-se relevante o esclarecimento de todos os mecanismos relacionados a este fenômeno. Assim, o presente estudo teve como objetivo comparar o desempenho muscular do Q e IT de idosos assintomáticos e portadores de OA de joelhos e correlacioná-los com a dor e o edema presentes na articulação comprometida.

\section{METODOLOGIA}

\section{Amostra}

Participaram deste estudo 15 voluntários com diagnóstico clínico e radiográfico de OA bilateral de joelho, segundo os critérios do American College of Rheumatology ${ }^{2}$, com idade entre a 65 e 80 anos que viviam na comunidade e possuíam marcha independente (aquela realizada sem auxílio externo) ${ }^{12}$. Também participaram deste estudo, 15 idosos assintomáticos que preencheram os critérios de inclusão previamente mencionados no que concerne à idade e independência da marcha, além de ausência de quaisquer sinais e sintomas clínicos de OA nos joelhos. Os idosos foram recrutados de grupos sociais e centros de atendimentos à saúde de Belo Horizonte e também atenderam aos seguintes critérios de exclusão: presença de prótese total ou parcial de quadris e/ ou joelhos, doenças neurológicas, outras doenças reumáticas, déficit cognitivo que comprometesse a compreensão dos testes, amplitude de movimento menor que $90^{\circ}$ de flexão dos quadris e amplitude de movimento abaixo de $90^{\circ}$ de flexão e $5^{\circ}$ de extensão dos joelhos. Os mesmos foram alocados em dois grupos: grupo de idosos assintomáticos (grupo AS) e grupo de idosos com OA de joelho (grupo OA). Na formação dos grupos os indivíduos foram pareados por sexo e idade.

Este estudo foi aprovado pelo Comitê de Ética em Pesquisa da Universidade Federal de Minas Gerais e os participantes assinaram termo de consentimento livre esclarecido assegurando seus direitos de acordo com a resolução N 196/96 do Conselho Nacional de Saúde.

\section{Instrumentação}

Para avaliação da performance muscular de IT e Q foi utilizado o dinamômetro isocinético Biodex System 3 Pro (Biodex Medical Systems, Shirley, New York) ${ }^{13}$.

Para registro da dor no joelho, foi utilizada a Escala Visual Analógica (EVA) numérica. Este instrumento constitui-se de uma linha reta horizontal com $10 \mathrm{~cm}$ de comprimento, numerada de 0 a 10 a cada centímetro, na qual suas terminações são definidas como os limites extremos da sensação dolorosa ${ }^{14}$.

\section{Procedimento}

No grupo OA os dados foram coletados do membro mais sintomático e no grupo AS a coleta foi realizada no membro corresponde à dominância de seu par. Foi considerada como a perna dominante, aquela escolhida pelo indivíduo para chutar uma bola ${ }^{12}$.

Inicialmente, os participantes foram submetidos a uma avaliação que constou de dados como idade, sexo, massa corporal, estatura, índice de massa corporal (IMC), membro inferior dominante e mais sintomático, presença e intensidade 
de dor, presença de edema, nível de atividade física (NAF) e grau de acometimento da OA. A classificação radiográfica da OA seguiu os critérios propostos por Kellgren e Lawrence ${ }^{15}$.

Em seguida, para aquecimento, seguindo protocolo utilizado com idosos, os participantes pedalaram em bicicleta ergométrica por 5 minutos em velocidade confortável ${ }^{8}$.

Para realização do teste de performance dos músculos $\mathrm{Q}$ e IT, os participantes foram posicionados sentados no dinamômetro isocinético com o tronco, pelve e coxa estabilizados por cintos e o eixo foi alinhado com o epicôndilo lateral do fêmur ${ }^{13}$. Após o posicionamento, os voluntários praticaram três repetições submáximas, como forma de familiarização. Posteriormente, foram dados 20 segundos de descanso, e, então, foi realizada a avaliação da performance muscular com 5 repetições na velocidade de $60 \%$, na ADM de $5^{\circ}$ a $90^{\circ}$ de flexão do joelho ${ }^{16}$. Durante a realização dos testes isocinéticos foi dado o mesmo estímulo verbal a todos os participantes para obtenção de força máxima ${ }^{13}$. A calibração do equipamento, bem como a correção da gravidade em todos os testes, foram executados conforme instrução do fabricante e o mesmo foi operado por avaliador experiente.

\section{Redução dos Dados}

Os parâmetros trabalho do quadríceps (TRAB Q) e trabalho dos isquiotibiais (TRAB IT) normalizados pela massa corporal, obtidos através do teste de desempenho muscular isocinético, bem como a relação do pico de torque IT/Q (Relação IT/Q), foram analisados.

O NAF de cada participante foi classificado de acordo com o preconizado pelo Americam College of Sports Medicine (ACSM), que considera ativo aquele que pratica atividade física moderada, por no mínimo 30 minutos na maioria dos dias da semana ${ }^{17}$.

A presença de edema foi avaliada como descrito por Silva, pressionando-se, as bolsas suprapatelar e infrapatelar com a comissura digital do polegar e do indicador. Com os dedos indicador e médio, pressionou-se a região medial do joelho e, após retirada a pressão, observou-se se esta sofreu um abaulamento, o que indica a presença de edema ${ }^{18}$.

\section{Análise Estatística}

Foi realizada análise descritiva das variáveis idade, massa corporal, estatura e IMC.

Teste $t$-Student para amostras independentes foi utilizado para concluir sobre as diferenças entre os grupos quanto às médias das variáveis demográficas, antropométricas, TRAB Q, TRAB IT e Relação IT/Q. Previamente, a normalidade da distribuição da amostra foi testada através do teste de Kolmogorov-Smirnov, que mostrou valor de p> 0,10 em todas as análises indicando normalidade na distribuição dos dados.

Para realizar a comparação do NAF entre os grupos foi usado o teste Qui-quadrado.
O coeficiente de Spearman foi calculado para verificação da correlação entre as variáveis edema e dor com as variáveis TRAB Q, TRAB IT e Relação IT/Q.

Estabeleceu-se nível de significância de $\alpha=0,05$ para todas as análises estatísticas.

\section{RESULTADOS}

Em ambos os grupos, 11 voluntários (73,3\%) pertenciam ao sexo feminino. A média da idade dos participantes do grupo AS foi de 71,33 $\pm 3,99$ anos (65 a 79) e do grupo OA foi de 71,47 $\pm 3,93$ anos (65 a 79). O IMC teve média de $26,84 \pm 4,92 \mathrm{~kg} / \mathrm{m}^{2}$ no grupo AS e $29,48 \pm 4,41 \mathrm{~kg} / \mathrm{m}^{2}$ no grupo OA.

Quanto ao índice de gravidade do exame radiológico, predominou o grau III, com sete voluntários (46,7\%), seguido do grau II, com seis casos (40,0\%).

Dentre os participantes do grupo OA, dez (66,5\%) possuíam edema e onze (73,3\%) apresentaram dor. A pontuação da dor na EVA variou de zero a sete, com predomínio das pontuações zero e três pontos, relatadas por quatro participantes e dois pontos relatadas por três voluntários. No grupo AS, estas características clínicas estavam ausentes em 100\% de seus integrantes.

A comparação das variáveis idade, massa corporal, estatura e IMC entre os grupos, mostrou não haver diferença estatisticamente significante entre estas medidas $(p>0,05)$.

Para a variável NAF foi utilizado o Teste Qui-quadrado e também não houve diferença estatisticamente significativa entre os grupos $(\mathrm{p}=0,256)$ (Tabela 1$)$.

Tabela 1. Comparação do Nível de Atividade Física.

\begin{tabular}{ccccc}
\hline NAF* & \multicolumn{3}{c}{ Grupo } & Total \\
& & OA** $^{* *}$ & AS*** \\
\hline Ativo & $\mathrm{n}$ & 8 & 11 & 19 \\
& Porcentagem & $42,1 \%$ & $57,9 \%$ & $100,0 \%$ \\
Sedentário & $\mathrm{n}$ & 7 & 4 & 11 \\
& Porcentagem & $63,6 \%$ & $36,4 \%$ & $100,0 \%$ \\
Total & $\mathrm{n}$ & 15 & 15 & 30 \\
& Porcentagem & $50,0 \%$ & $50,0 \%$ & $100,0 \%$ \\
\hline
\end{tabular}

*Nível de atividade física segundo critérios do ACSM; **Com osteoartrite; *** Assintomáticos; $\mathrm{p}=0,256$ (Teste Qui-quadrado).

A variável TRAB Q apresentou diferença estatisticamente significativa ( $\mathrm{p}=0,001)$, sendo o valor médio desta variável maior para o Grupo AS. Em se tratando da variável TRAB IT, embora menor no grupo OA, a diferença não foi estatisticamente significativa ( $\mathrm{p}>0,05)$. Quanto a Relação IT/ $\mathrm{Q}$, verificou-se diferença estatisticamente significativa entre 
os grupos $(\mathrm{p}=0,04)$, sendo o valor médio maior para o grupo OA (Tabela 2).

O Coeficiente de Spearman rho revelou não haver correlação estatisticamente significativa entre as variáveis dor e edema com as medidas TRAB Q, TRAB IT e Relação $\mathrm{IT} / \mathrm{Q}$ (rho $\leq-0,468 \mathrm{p} \geq 0,229$ ).

Tabela 2. Comparação das variáveis TRAB Q, TRAB IT e RELAÇÃO IT/Q.

\begin{tabular}{cccccc}
\hline Variável & Grupo & Média & DP** & T*** & Valor-p \\
\hline TRAB Q* & OA & 106,853 & 27,3665 & $-3,782$ & $\mathbf{0 , 0 0 1}$ \\
& AS & 147,400 & 31,2346 & & \\
TRAB IT $^{*}$ & OA & 59,187 & 19,2416 & $-1,674$ & 0,105 \\
& AS & 71,953 & 22,4048 & & \\
Relação IT/Q* $^{*}$ & OA & 51,180 & 11,2240 & 2,154 & $\mathbf{0 , 0 4 0}$ \\
& AS & 43,667 & 7,5149 & & \\
& & & & &
\end{tabular}
*Valores obtidos no dinamômetro isocinético a $60 \%$ s; ** Desvio padrão;
***Teste $t$ - Student.

\section{DISCUSSÃO}

$\mathrm{Na}$ amostra estudada, o grupo AS apresentou IMC de $26,84 \mathrm{~kg} / \mathrm{m}^{2}$ e o OA $29,48 \mathrm{~kg} / \mathrm{m}^{2}$ e, segundo os critérios de caracterização do estado nutricional da população idosa, sugeridos por Lipschitz ${ }^{19}$, IMC $>27 \mathrm{~kg} / \mathrm{m}^{2}$ determina o sobrepeso. Portanto os idosos do grupo OA se enquadravam na faixa de sobrepeso.

Uma vez que a literatura aponta a influência do sexo, idade, medidas antropométricas e NAF no desempenho muscular ${ }^{20}$, foram realizadas análises das diferenças destas variáveis entre os grupos do estudo. Assim, a análise das variáveis sexo (73,3\% de sexo feminino em ambos os grupos), idade (OA 71,47 $\pm 3,93$ anos e AS 71,33 $\pm 3,99$ anos), bem como das variáveis antropométricas e NAF não demonstrou diferenças estatisticamente significantes ( $\mathrm{p}>$ $0,05)$ entre os grupos. Em suma, estes dados vêm caracterizar a homogeneidade de ambos os grupos em relação a essas variáveis, o que possibilitou as comparações propostas nesta pesquisa.

O parâmetro trabalho muscular nos informa sobre a força que o músculo ou grupo muscular pode desenvolver durante toda a incursão do movimento articular, o que pode ser computado como a área sob a curva de torque ${ }^{13}$. Já o pico de torque refere-se à força máxima em um ponto específico da ADM percorrida. Desta forma, a análise do parâmetro trabalho nos fornece uma informação mais ampla do desempenho muscular que o pico de torque e, por isso, foi eleito para análise neste estudo. Não obstante, analisando a literatura que contempla o desempenho muscular em idosos, constatou-se uma predominância na utilização do parâmetro pico de torque em detrimento do trabalho e dos demais parâmetros ${ }^{21}$, fato que dificulta as comparações da literatura com os resultados do presente estudo.

Verificou-se, neste estudo, uma redução significativa do TRAB Q no grupo OA, porém esta redução não guardou a mesma proporção para o TRAB IT. Estes achados estão de acordo com os encontrados por outros estudos ${ }^{7,20}$ e são independentes da massa corporal dos participantes.

A fraqueza preferencial da musculatura extensora sugere que a atrofia por desuso não é o principal responsável por este resultado, principalmente porque não houve diferença significativa no NAF segundo os critérios do ACSM entre os grupos OA e AS. Estudo realizado por Slemenda et al. ${ }^{7}$ no qual a massa magra foi quantificada em quilogramas, constatou que até mesmo os indivíduos com OA de joelho que possuíam igual ou maior massa muscular apresentaram menor desempenho muscular. Este fenômeno, foi atribuído a IMA que parece estar relacionado à presença de edema ${ }^{9}$ e de dor ${ }^{20,22}$ articulares. Neste caso, ocorreria um input aferente alterado para a articulação afetada, o que, conseqüentemente, acarretaria em uma redução no estímulo eferente do motoneurônio do Q, influenciando negativamente o seu desempenho ${ }^{9,10}$.

Contudo, neste estudo, o edema e a dor não mostraram uma correlação significativa com as variáveis TRAB Q e IT e Relação IT/Q. Talvez isto possa ser atribuído ao tamanho reduzido da amostra. Além disso, o valor predominante relatado para dor foram três e dois, ou seja, uma intensidade relativamente baixa em uma escala de zero a dez. Correlação significativa entre estas variáveis foi previamente reportada nos estudos de Stauffer et al. ${ }^{23}$ e Teixeira e Olney ${ }^{20}$. Estudo de McNair et al. ${ }^{9}$, que teve como objetivo investigar o efeito do excesso de fluído no interior da articulação do joelho na capacidade de geração de torque pelo músculo $\mathrm{Q}$ e determinar se ele pode ser alterado por exercício desta articulação, verificou que o torque diminui com a presença de fluido intraarticular excessivo. Também puderam verificar, neste mesmo estudo, que o torque aumentou após exercícios submáximos ${ }^{9}$. Segundo os autores, talvez este último achado mostre que exercícios submáximos aumentam a complacência da cápsula implicando em redução das descargas subseqüentes dos mecanorreceptores nela localizada ${ }^{9}$. Pressuposto este que reforça o princípio da IMA.

A Relação IT/Q é um parâmetro comumente usado para descrever o equilíbrio muscular da articulação do joelho ${ }^{24}$, e uma alteração da mesma pode predispor tanto a articulação quanto o grupo muscular mais fraco à lesão ${ }^{13}$. Nos resultados deste estudo, a relação IT/Q apresentou-se significativamente aumentada no grupo OA em comparação com o AS. Isto se deu pelo fato já mencionado anteriormente de uma menor 
força ter sido desenvolvida pelo Q em relação à força dos IT relativamente preservada.

Estudo de Dias et al. ${ }^{16}$ que teve como objetivo estabelecer valores normativos para a relação IT/Q de idosas saudáveis, encontrou um valor médio de $47,95 \%$ na velocidade de $60^{\circ} / \mathrm{s}^{16}$. Confrontando este achado com o do grupo AS do presente estudo (valor médio de 43,67\%), constata-se valor menor que o por eles preconizado. Esta disparidade, possivelmente, se atribui à idade média mais avançada e à presença de indivíduos do sexo masculino no presente estudo.

Teixeira e Olney ${ }^{20}$ realizaram a medida da relação IT/ $\mathrm{Q}$ em dois grupos de idosos, o primeiro com OA de joelhos e o segundo assintomático, e encontraram valores de $72 \%$ e $53 \%$ respectivamente ${ }^{20}$. Apesar do dinamômetro isocinético utilizado ter sido de marca diferente, comparando os resultados de Teixeira e Olney com os deste estudo verificou-se que o grupo com OA daquele, também apresentou um valor maior para esta medida em comparação com seus pares.

A diminuição preferencial da ativação do grupo extensor do joelho também pode estar relacionada ao fato de, na presença do edema nesta articulação, a posição de maior conforto ser em flexão, a qual corresponde à posição destravada desta articulação ${ }^{25}$. Desta forma, supõe-se que a não extensão seria uma estratégia antálgica gerada pela inibição do Q.

Uma vez que técnicas metodológicas podem influenciar nos resultados da performance muscular no dinamômetro isocinético, os seguintes parâmetros para utilização deste instrumento foram rigorosamente respeitados: a prévia calibração do equipamento, o posicionamento cuidadoso dos participantes, a correção da gravidade, a familiarização com o teste, o fornecimento de adequadas orientações sobre o teste e de estímulo verbal visando à motivação, além da realização de prévio aquecimento dos músculos testados. Para minimizar possíveis substituições, o tronco, pélvis e coxa foram devidamente estabilizados ${ }^{13}$.

\section{CONCLUSÃO}

O déficit no desempenho muscular de idosos portadores de OA de joelho é uma característica comum nesta população. $\mathrm{Na}$ amostra estudada (grupo OA e grupo AS) este fenômeno acometeu preferencialmente o grupo muscular extensor embora ambos os grupos apresente semelhante NAF. Este fato nos leva a concluir que a redução da força muscular nesta população não deve ser atribuída, unicamente, ao menor NAF por ela adotado. Além disso, embora, pesquisas apontem para uma possível associação do decréscimo no desempenho muscular com a dor e o edema manifestados nesta doença ${ }^{17,31,38}$, o presente estudo não corroborou este fato, o que, talvez, possa ser atribuído ao tamanho relativamente pequeno desta amostra.
Diante destas conclusões, este estudo sugere que a intervenção fisioterapêutica visando à melhoria do desempenho muscular de portadores de OA de joelho não pode basearse, exclusivamente, na mudança comportamental através da implementação de um maior nível de atividade física destes pacientes. Sugere ainda a realização de futuras pesquisas para elucidar a existência ou não de correlação entre disfunção muscular e presença da dor e do edema em portadores de OA de joelhos. Para tanto, sugere-se a execução de estudos com uma população maior, bem como com outras faixas etárias. Também sugere-se a utilização de instrumento que meça o edema de forma quantitativa e precisa.

\section{REFERÊNCIAS BIBLIOGRÁFICAS}

1. Martin DF. Pathomechanics of knee osteoarthritis. Med Sci Sports Exerc 1994; 26(12):1429-1434.

2. Altman R, Asch E, Bloch D, Bole G, Borenstein D, Brandt K, et al. Development of criteria for the classification and reporting of osteoarthritis. Classification of osteoarthritis of the knee. Diagnostic and Therapeutic Criteria Committee of the American Rheumatism Association. Arthritis Rheum 1986; 29(8):10391049.

3. Felson DT, Zhang Y, Hannan MT, Naimark A, Weissman BN, Aliabadi P, et al. The incidence and natural history of knee osteoarthritis in the elderly. The Framingham Osteoarthritis Study. Arthritis Rheum 1995; 38(10):1500-1505.

4. Frontera WR, Hughes VA, Fielding RA, Fiatarone MA, Evans WJ, Roubenoff R. Aging of skeletal muscle: a 12-yr longitudinal study. J Appl Physiol 2000; 88(4):1321-1326.

5. Evans WJ. Effects of exercise on senescent muscle. Clin Orthop 2002;(403 Suppl):S211-S220.

6. Patten C, Craik RL. Sensorimotor changes and adaptation in the older adult. In: Guccione AA, editor. Geriatric Physical Therapy. Mosby: St Louis, 2000: 78-109.

7. Slemenda C, Brandt KD, Heilman DK, Mazzuca S, Braunstein EM, Katz BP, et al. Quadriceps weakness and osteoarthritis of the knee. Ann Intern Med 1997; 127(2):97-104.

8. Schilke JM, Johnson GO, Housh TJ, O’Dell JR. Effects of muscle-strength training on the functional status of patients with osteoarthritis of the knee joint. Nurs Res 1996; 45(2):68-72.

9. McNair PJ, Marshall RN, Maguire K. Swelling of the nee Joint: Effects of Exercise on Quadriceps Muscle Strength. Arch Phys Med Rehabil 1996; 77:896-899.

10. Rutherford OM, Jones DA, Newham DJ. Clinical and experimental application of the percutaneous twitch superimposition technique for the study of human muscle activation. J Neurol Neurosurg Psychiatry 1986; 49(11):1288-1291.

11. Guccione AA. Arthritis and the process of disablement. Phys Ther 1994; 74(5):408-414.

12. Sharma L, Hayes KW, Felson DT, Buchanan TS, Kirwan-Mellis G, Lou C, et al. Does laxity alter the relationship between strength and physical function in knee osteoarthritis? Arthritis Rheum 1999; 42(1):25-32. 
13. Perrin DH. Isokinetic Exercise and Assessment. Champaign, IL: Human Kinetics Publishers, 1993.

14. Bolognese JA, Schnitzer TJ, Ehrich EW. Response relationship of VAS and Likert scales in osteoarthritis efficacy measurement. Osteoarthritis Cartilage 2003; 11(7):499-507.

15. Kellgren JH, Lawrence JS. Radiological assessment of osteo-arthrosis. Ann Rheum Dis 1957; 16(4):494-502.

16. Dias JMD, Arantes PMM, Alencar MA, Faria JC, Machala CC, Camargos FFO, et al. Relação Isquiotibiais/ Quadríceps em Mulheres Idosas Utilizando o Dinamômetro Isocinético. Rev Bras Fisioter 2004; 8(2):111-115.

17. Matsudo V, Matsudo S, Andrade D, Araujo T, Andrade E, de Oliveira LC et al. Promotion of physical activity in a developing country: the Agita Sao Paulo experience. Public Health Nutr 2002; 5(1A):253-261.

18. Silva OL. Exame Clínico do Joelho e da Perna. Semiologia do Aparelho Locomotor. Rio de Janeiro: Guanabara Koogan, 2003: 222-241.

19. Lipschitz DA. Screening for nutritional status in the elderly. Prim Care 1994; 21(1):55-67.
20. Teixeira LF, Olney SJ. Avaliação Clínica, Radiológica e Estudo Isocinético da Força Muscular em Pacientes Idosos Portadores de Osteoartrite (OA) do Joelho. Rev Fisioter Univ São Paulo 1995; 2(2):56-64.

21. Huang MH, Lin YS, Yang RC, Lee CL. A comparison of various therapeutic exercises on the functional status of patients with knee osteoarthritis. Semin Arthritis Rheum 2003; 32(6):398406.

22. O’Reilly SC, Jones A, Muir KR, Doherty M. Quadriceps weakness in knee osteoarthritis: the effect on pain and disability. Ann Rheum Dis 1998; 57(10):588-594.

23. Stauffer RN, Chao EYS, Györy AN. Biomechanical Gait Analysis of the Diseased Knee Joint. Biomechanical Gait Analysis 1977; 126:246-255.

24. Aagaard P, Simonsen EB, Magnusson SP, Larsson B, DyhrePoulsen P. A new concept for isokinetic hamstring: quadriceps muscle strength ratio. Am J Sports Med 1998; 26(2):231-237.

25. Norkin C, Levangie PK. Joint Structure and Function: A Comprehensive Analysis. Philadelphia: P.A. Davis Company, 2001. 